\title{
Glottopol
}

Revue de sociolinguistique en ligne

$33 \mid 2020$

Varia - Liberté, égalité, diversité !

Normes interactionnelles globalisées et

communautés de pratique discontinues : les dynamiques sociolinguistiques du tourisme international

\section{Adam Wilson}

\section{OpenEdition}

\section{Journals}

Édition électronique

URL : https://journals.openedition.org/glottopol/604

DOI : 10.4000/glottopol.604

ISSN : 1769-7425

Éditeur

Presses universitaires de Rouen et du Havre

Référence électronique

Adam Wilson, « Normes interactionnelles globalisées et communautés de pratique discontinues : les dynamiques sociolinguistiques du tourisme international », Glottopol [En ligne], 33 | 2020, mis en ligne le 01 janvier 2020, consulté le 02 octobre 2021. URL : http://journals.openedition.org/glottopol/604 ; DOI : https://doi.org/10.4000/glottopol.604 




\section{GLOTTOPOL}

Revue de sociolinguistique en ligne $n^{\circ} 33$ - février 2020

Varia : Liberté, égalité, diversité !

\section{SOMMAIRE}

Hommages à Jean-Baptiste Marcellesi : À ringrazià vi, par Niculau Sorba ; “Ciao Ziu ! » par Philippe Blanchet.

Clara Mortamet : Présentation du numéro : Liberté, égalité, diversité !

Manon Him-Aquilli : "Prendre la parole sans prendre le pouvoir ». La mise en registre de l'horizontalité dans les assemblées générales anarchistes/autonomes.

Siham Hocini : La formulation du désaccord dans les débats télévisés algériens : quels "effetsde-politesse "?

Pauline Rannou : Trajectoires de la surdité en France. Parents entendants d'enfants sourds : questionner la fragmentation de l'identité sourde.

Catherine Combaz-Champlaine: Les rectifications orthographiques de 1990 comme révélateurs du rapport des enseignants à l'orthographe.

Marie-Madeleine Bertucci : Éléments d'épistémologie pour l'étude des espaces ségrégués des banlieues urbaines sensibles de la région parisienne.

Élise Gandon: Usages du numérique et illectronisme: nouvelles tâches pour le lecteurscripteur, nouveaux enjeux d'apprentissage.

Marc Arabyan : Quelle langue pour la recherche neurolinguistique française?

José R. de Arellano : Le rapprochement entre l'espagnol et le portugais pendant la décennie dorée sud-américaine : une problématique de l'horizontalité supercentrale.

Adam Wilson: Normes interactionnelles globalisées et communautés de pratique discontinues : les dynamiques sociolinguistiques du tourisme international.

Mélanie Lancien : Caractérisation de la variation liée à la situation de communication : apport de l'acoustique à la phonostylistique.

Assémou Maurice Ludovic Assémou : La prononciation du français parlé en Côte d'Ivoire. 


\title{
NORMES INTERACTIONNELLES GLOBALISÉES ET COMMUNAUTÉS DE PRATIQUE DISCONTINUES : LES DYNAMIQUES SOCIOLINGUISTIQUES DU TOURISME INTERNATIONAL ${ }^{1}$
}

\author{
Adam Wilson \\ Université de Lorraine, IDEA
}

\section{Introduction}

Nous vivons à l'ère de la globalisation. Au départ un processus d'ouverture des marchés financiers, cette idéologie de dérégulation s'étend aujourd'hui à d'innombrables aspects de la vie sociale. La globalisation se présente comme un processus d'expansion mondiale de l'idéologie néolibérale, entrainant des transformations économiques, géographiques, sociales et culturelles dans lesquelles tout devient marchandise. La mobilité est le nerf de cette transformation : de plus en plus de capitaux, de produits, d'idées, d'informations et, surtout, d'êtres humains traversent de plus en plus de frontières nationales au service du marché mondial (Urry, 2007). Même si la mobilité humaine à grande échelle n'a rien de nouveau, les phénomènes de mobilité liés à la globalisation, facilités par le développement récent des transports, sont d'une ampleur inédite. Ajoutons à cela les innovations en termes de technologies de la communication, et la résultante est un changement social et philosophique profond où l'espace-temps se voit transformé (Inda \& Rosaldo, 2007). Pour la première fois dans l'histoire, le monde est conçu comme un seul et unique espace social (Scholte, 2002).

Les spécialistes de la sociolinguistique ont su pressentir qu'un tel changement social aurait nécessairement d'importants corrélats socio-langagiers. En effet, les dernières années ont vu une explosion de travaux qui présentent, d'une part, l'apparition de nouvelles formes et pratiques langagières en lien avec les transformations socio-économiques de la globalisation, et d'autre part, le rôle joué par le langage dans les processus de construction de la globalisation - ainsi que les implications possibles sur le sort des individus concernés (cf. Blommaert, 2010 ; Coupland, 2010 ; Heller \& Duchêne, 2012 ; Auzanneau \& Trimaille, 2017, par ex.). Ces travaux ont permis d'identifier un certain nombre de situations clés pour l'exploration des enjeux sociolinguistiques de la globalisation, telles que les flux de migration, les entreprises multinationales, les villes globales ou les institutions internationales.

\footnotetext{
${ }^{1}$ Certaines parties de cette recherche ont été effectuées pendant mon doctorat entrepris au Laboratoire Parole et Langage (Aix-Marseille Université). Le travail de terrain a été rendu possible grâce au soutien technique du Centre d'expérimentation sur la parole du LPL.
} 
Parmi les situations clés, le tourisme a été souligné comme étant particulièrement pertinent puisqu'il constitue à la fois un produit et un moteur de la globalisation, reflétant ainsi les dynamiques socio-économiques qui la sous-tendent (Jaworski \& Thurlow, 2010a). Le tourisme en tant qu'industrie repose sur deux pierres angulaires de la globalisation : la commodification (ou marchandisation) et la mobilité. Il n'est donc pas étonnant de constater une augmentation récente des recherches sociolinguistiques menées sur le tourisme, explorant entre autres le discours du tourisme (Dann, 1996 ; Jaworski \& Thurlow, 2010b), le tourisme comme lieu de commodification du langage (Kelly-Holmes \& Pietikäinen, 2014 ; Heller et al., 2014) et montrant même comment le langage et le discours contribuent à l'élaboration de l'expérience touristique en elle-même (Thurlow \& Jaworski, 2011). L'ensemble de ces travaux mettent également l'accent sur le tourisme comme un domaine de consommation et de commodification qui contribue au maintien, voire à l'accentuation, des dynamiques de privilège et d'inégalités sociales liées à la globalisation.

Malgré la valeur inestimable de ces travaux, ils ont eu tendance à s'intéresser aux sources textuelles ou médiatisées, ce qui crée presque automatiquement une focalisation sur l'avant et l'après de l'expérience touristique ainsi que sur le langage écrit et d'autres formes sémiotiques visuelles. Très peu de travaux abordent donc le pendant de l'expérience touristique ou les interactions que les touristes ont au sein de celle-ci. Or les touristes, individus mobiles dans une situation de commodification, sont l'incarnation par excellence des processus de la globalisation - d'où l'intérêt de se pencher sur les interactions auxquelles ils prennent part.

Cet article a donc pour objectif d'explorer un contexte de tourisme international par le biais de l'étude des interactions en face-à-face. Il s'agit d'examiner le comportement linguistique dans un cadre emblématique de la globalisation afin de tisser des liens entre les dynamiques langagières observées et les dynamiques socio-économiques plus larges du tourisme et de la globalisation. Dans un premier temps, nous abordons les changements sociaux et linguistiques liés à la globalisation et montrons en quoi le tourisme constitue un contexte clé de celle-ci. Puis, nous présentons le contexte de l'étude et la méthodologie de la recherche. Ensuite, l'analyse des données se décline en trois parties. Tout d'abord, nous identifions un certain nombre de caractéristiques de la situation étudiée qui pourraient mener à son identification en tant que « situation de parole globalisée ». Ensuite, nous dégageons des schémas de comportement dans les interactions qui nous permettent d'identifier certaines normes interactionnelles. Enfin, en partant de l'observation de ce comportement normatif, nous proposons la notion de « communauté de pratique discontinue » afin de rendre compte de la socialisation des locuteurs à ces normes. Nous terminons cet article par une discussion sur les répercussions sociales des dynamiques sociolinguistiques abordées avant de proposer une courte conclusion.

\section{Globalisation : changements sociaux, changements linguistiques}

Le processus extrêmement vaste et complexe que constitue la globalisation mérite d'être clarifié et défini avant toute tentative d'analyse sociolinguistique. Nous avons choisi d'employer ici le mot "globalisation » plutôt que «mondialisation », car certains chercheurs ont souligné le fait que ce dernier terme a tendance à se limiter aux aspects géographiques des transformations observées, tandis que la notion de globalisation inclut également des aspects sociaux, culturels et économiques (cf. Selim, 2004, par exemple). Bien que très difficile à délimiter (cf. Lecler, 2013, pour une discussion détaillée), la notion de globalisation peut être définie comme un processus d'expansion mondiale des politiques, pratiques et idéologies du néolibéralisme (Scholte, 2005). Au départ, il s'agissait d'une doctrine économique, propageant l'idéologie du libre-marché et conduisant à la dérégulation des marchés intranationaux comme internationaux (cf. Plihon, 2007). Plus récemment, ce même ensemble de politiques, pratiques 
et idéologies s'est étendu à d'autres marchés de produits et de services, créant progressivement un seul et unique marché mondial, ignorant les frontières nationales, et sur lequel tout type de marchandise peut être échangé (Lecler, 2013).

L'élément central de ces échanges est la mobilité internationale : les capitaux, biens et personnes doivent pouvoir circuler partout dans le monde afin de faire partie de ce marché global. Les êtres humains sont donc de plus en plus mobiles, que ce soit pour leur travail, leurs loisirs ou d'autres raisons personnelles et professionnelles. La mobilité, que ce soit dans la migration, le tourisme ou les programmes universitaires d'échange international (pour ne nommer que quelques exemples), est au cœur de nos vies contemporaines (Urry, 2007).

En termes d'impact sur le langage et les pratiques linguistiques, on peut dire que la mobilité qui caractérise la globalisation crée de plus en plus de "points de rencontre » des individus mobiles, ce que Romain Lecler (2013 : 49) appelle des « nœuds du réseau » de la globalisation. Ces nœuds constituent des situations de contact de langues caractérisées par une diversité accrue de locuteurs et de répertoires linguistiques.

La sociolinguistique, en tant que discipline qui interroge les rapports entre dynamiques sociales et dynamiques linguistiques, occupe une place particulièrement intéressante pour étudier ces situations inédites de contacts de langues. Ainsi, l'étude des effets de l'intensification de la mobilité sur les pratiques langagières a mené au développement de plusieurs concepts afin de rendre compte de la visibilité des parcours de mobilité dans certaines pratiques langagières, tels que la superdiversity (Arnaut et al., 2015), le translanguaging (Garcia \& Wei, 2013) ou le metrolingualism (Pennycook \& Otsuji, 2015). En outre, certains travaux ont abordé les changements de valeurs des ressources linguistiques au sein du nouveau marché mondial (Duchêne \& Heller, 2012). Ces recherches mettent en avant les dynamiques de commodification linguistique observables dans de nombreux contextes globalisés (Heller, 2010) et la place de plus en plus importante du langage au sein du travail, créant des «travailleurs du langage » (Boutet, 2008) et menant à une conception du langage comme habileté monnayable imbibée de capital symbolique (Duchêne, 2012).

Les recherches en sociolinguistique ont également identifié un certain nombre de contextes particulièrement fertiles pour l'analyse des liens entre les transformations socio-économiques de la globalisation et les transformations des pratiques langagières, tels que les flux migratoires, le commerce international et le tourisme.

\section{Tourisme international : questions sociolinguistiques}

Malgré son ampleur en tant que phénomène social, l'apparition du tourisme dans les recherches en sociolinguistique, et en sciences humaines et sociales plus généralement, fut relativement tardive. La première monographie abordant les aspects sociolinguistiques du tourisme est proposée par Graham Dann en 1996 (Dann, 1996). En s'inspirant des concepts mis en avant dans des études sur le tourisme en sociologie, géographie et anthropologie - à savoir, l'authenticité, l'étrangeté, la récréation et le conflit - Dann expose comment ils se manifestent dans le discours du tourisme à travers une étude discursive de matériels textuels tels que des publicités, des prospectus et des reportages.

Environ une décennie plus tard, Adam Jaworski et Crispin Thurlow reprennent le flambeau et initient une période prolifique de publications sur le tourisme en analyse du discours et en sociolinguistique, qui se poursuit encore aujourd'hui. Les auteurs approfondissent la réflexion sur l'élaboration discursive de l'authenticité et de l'étrangeté dans des textes et d'autres médias touristiques (Jaworski \& Thurlow, 2010b) et s'intéressent également aux questions d'identité et de performance en milieu touristique (Jaworski \& Thurlow, 2009). Ils montrent que les aspects discursifs de la performance de certaines identités en milieu touristique contribuent de 
façon importante à l'élaboration de l'expérience touristique elle-même (Jaworski \& Thurlow, 2010a).

Deux thématiques spécifiques développées par Jaworski, Thurlow et d'autres auteurs méritent une attention particulière. D'une part, de nombreux travaux exposent des processus de commodification linguistique en milieu touristique, que ce soit la transformation du langage en « marchandise » ou l'utilisation du langage au service de la commodification d'autres produits (Kelly-Holmes \& Pietikäinen, 2014 ; Heller et al., 2014, entre autres). D'autre part, Thurlow et Jaworski (2013) montrent en quoi le tourisme constitue une mobilité d'élite, accessible seulement à ceux qui bénéficient de la globalisation. Cette observation les pousse à identifier le milieu touristique comme étant un milieu de "globalisation banale » (Jaworski \& Thurlow, 2010a) dans lequel des dynamiques d'inégalité sociale de la globalisation sont produites sans que les acteurs s'en rendent compte.

La notion de "globalisation banale" peut être mise en lien avec l'idée des milieux touristiques comme étant des "nœuds" dans les réseaux de la globalisation. Le milieu touristique constitue un contexte dans lequel des locuteurs (et leurs langues) mobiles incorporent et produisent les dynamiques de la globalisation, et peut être envisagé à la fois comme reflet et moteur de ces dernières. Les interactions touristiques représentent donc un élément crucial dans l'élaboration de la globalisation, charnière entre des contextes occasionnels et des dynamiques sociales beaucoup plus larges. Cependant, malgré l'identification de l'intérêt sociolinguistique de ces interactions par Cohen \& Cooper (1986) dans l'un des premiers articles consacrés au langage dans le tourisme, les interactions en milieu touristique n'ont reçu que très peu d'intérêt dans les recherches sociolinguistiques. Les interactions pendant les visites guidées constituent une exception importante. Cependant, ces analyses ont eu tendance à se focaliser sur l'orientation spatiale et gestuelle des interlocuteurs (De Stefani \& Mondada, 2014) ou les aspects discursifs des visites (Vitorio, 2014). En dehors de ces contributions importantes, très peu de recherches ont été entreprises sur les interactions spontanées en milieu touristique, alors qu'elles semblent offrir une fenêtre privilégiée pour l'observation des processus d'une globalisation « banale ».

Le but de cet article est donc d'étudier de telles interactions spontanées en face-à-face dans un lieu touristique. Nous avons comme objectif d'explorer les rapports entre les pratiques langagières dans ce contexte et des dynamiques socio-économiques (et langagières) plus larges liées au tourisme et à la globalisation. Pour ce faire, un contexte particulièrement pertinent pour l'étude des interactions dans une situation de tourisme international a été identifié : l'Office de Tourisme de Marseille, en France.

\section{À la recherche d'un contexte globalisé : l'Office de Tourisme de Marseille}

Marseille se présente comme une ville particulièrement intéressante pour l'étude du tourisme international. Bien qu'entourée par des destinations touristiques mondialement connues, la deuxième ville de France a longtemps souffert d'une mauvaise réputation. Depuis une vingtaine d'années, les autorités urbaines lancent de multiples projets ambitieux de rénovation urbaine afin de redorer l'image de la ville et inciter de nouvelles arrivées à Marseille, que ce soit des nouveaux habitants, entreprises ou touristes.

Le tourisme constitue un pilier de cette stratégie et la ville consacre beaucoup de ressources au développement de l'infrastructure touristique et à l'organisation d'événements mondiaux qui visent à attirer des visiteurs vers la cité phocéenne. Récemment, et notamment depuis la désignation de Marseille comme Capitale européenne de la culture en 2013, ces efforts ont commencé à porter leurs fruits (Peraldi et al., 2015). La ville a connu une augmentation 
importante du nombre de visiteurs nationaux et internationaux. Marseille devient donc un point de rencontre de flux de touristes, c'est-à-dire de flux de mobilités globalisées.

Ce statut de nouveau point de convergence pour les parcours de mobilité touristique (et ainsi globalisé) fait de Marseille un cadre idéal pour explorer les dynamiques sociolinguistiques de ceux-ci. L'Office de Tourisme et des Congrès de Marseille (désormais OdT) présente l'intérêt d'être à la fois le lieu central de l'élaboration de la stratégie touristique de Marseille et un lieu visité par un grand nombre de touristes. En outre, il s'agit d'une institution donnant lieu de par sa nature à un grand nombre d'interactions en face-à-face, que ce soit entre touristes ou entre les touristes et les employés de l'OdT. Ces interactions constituent donc un exemple d'échanges entre une population "mobile » et des membres d'une population "locale ». Ainsi, il s'agit d'un véritable exemple d'un nœud de réseaux de la globalisation, un lieu dans lequel des éléments mobiles interviennent de façon éphémère.

Marseille, et plus précisément l'OdT, constituent un contexte permettant de soulever des questions pertinentes pour la description et l'analyse des pratiques langagières dans un contexte emblématique de la globalisation. Quelles sont les effets sociolinguistiques des nouvelles formes de mobilité touristique à Marseille ? Quels rapports se tissent entre les dynamiques langagières observables à l'OdT et les dynamiques socio-économiques plus larges du tourisme et de la globalisation?

Afin de répondre à ces questions, nous adoptons une approche méthodologique et théorique ethnographique, permettant de mettre les comportements linguistiques observés à l'OdT en relation avec des éléments sociaux plus large. Nous avons entrepris un travail de terrain à l'OdT sur plusieurs périodes entre 2014 et 2016, un travail qui consistait à effectuer des observations, réaliser des entretiens formels et informels avec l'ensemble des acteurs de l'OdT et récolter des documents touristiques disponibles à l'OdT. Nous avons également élaboré le corpus MITo (Corpus Marseillais d'Interactions Touristiques, cf. Wilson, 2016), un corpus audio constitué de 93 enregistrements entièrement transcrits et annotés d'interactions entre des touristes internationaux et des conseillers touristiques qui travaillent aux guichets de l'OdT. Ces données forment la base de notre analyse et sont abordées à travers un cadre théorique qui nous permet à la fois d'analyser finement les pratiques langagières des locuteurs et de mettre en relation des faits linguistiques microscopiques avec leur contexte social plus vaste. Cet outillage réunit des éléments de la sociolinguistique interactionnelle (Gumperz, 1989), de la sociolinguistique critique (Heller 2003) et de la « sociolinguistique politique » (Canut et al., 2019).

Avant de pouvoir explorer les liens éventuels entre les dynamiques linguistiques et des dynamiques sociales plus larges dans ce contexte, il faut en premier lieu décrire les pratiques langagières que l'on peut observer à l'OdT. Les prochaines sections présentent les résultats de cette analyse. Tout d'abord, nous identifions certaines «particularités » du contexte observées pendant le travail ethnographique qui semblent être en rapport avec la nature globalisée des mobilités présentes à l'OdT. Puis, nous nous penchons sur les données interactionnelles et dégageons certains schémas de comportement normatif.

\section{Caractéristiques d'un contexte sociolinguistique globalisé}

Les premières observations du travail de terrain sont centrées sur les caractéristiques de la situation de parole en elle-même. L'OdT comprend cinq particularités qu'il semble primordial de prendre en compte afin de décrire et interpréter le comportement linguistique observé dans ce contexte.

Interactions fugaces. À l'OdT, nous observons ce que nous appelons, suite à Jaworski \& Thurlow (2010a), des « interactions fugaces ». Les interactions à l'OdT peuvent être qualifiées de «fugaces» puisque les interlocuteurs ne se connaissent pas - et, probablement, ne se 
connaitront jamais : l'échange à l'OdT constitue généralement la première et la dernière fois qu'ils vont interagir. Autrement dit, les participants dans ces interactions ne partagent aucune « histoire interactionnelle » (Vion, 1992), ce qui signifie que les locuteurs n'ont aucun indice sur lequel ils peuvent se baser pour orienter leur comportement linguistique.

Interactions en "tir rapide ». S'ajoute à la fugacité des interactions, un caractère «tir rapide $»$ : les interactions sont courtes ${ }^{2}$ et s'enchainent à brefs intervalles. Cette caractéristique peut être liée au nombre conséquent de touristes à renseigner par l'OdT, ce qui peut aller jusqu'à 3000 par jour pendant la période estivale, pour un maximum de quatre guichets d'information. Il existe ainsi une file d'attente quasi permanente et dès qu'un touriste a été renseigné et part du guichet, un autre touriste est prêt à prendre sa place immédiatement. Il n'est pas rare d'observer des conseillers qui parlent presque sans cesse, enchainant un grand nombre d'interlocuteurs. Bien entendu, chaque touriste présente un nouveau « défi » linguistique car les conseillers doivent activer des compétences d'adaptation pour ajuster leur comportement linguistique en très peu de temps de façon répétée.

Hétérogénéité. L'hétérogénéité des touristes, à la fois au niveau de leurs provenances et leurs répertoires langagiers, s'ajoute au caractère "tir rapide» pour complexifier la tâche des conseillers. Pendant les périodes d'observation, l'OdT a enregistré des visites d'individus de plus de 50 états différents, ce qui donne une indication de la diversité linguistique des visiteurs quand nous prenons en compte la variété possible de langues premières, de langues secondes, de dialectes ou de variétés présents.

Contexte sociolinguistique ambigu. Nous pourrions argumenter que tout contexte est sociolinguistiquement ambigu. Cependant, souvent, dans des situations « stables » sur le plan sociolinguistique, il existe une langue et/ou une variété non-marquée (Myers-Scotton, 1993) qui constitue un choix linguistique par défaut. Les nouvelles mobilités de la globalisation brouillent en quelque sorte ces schémas prévisibles et l'OdT en fournit un bon exemple. D'un côté, il s'agit d'une institution française située en France, ce qui suggère que le français serait clairement le choix linguistique non-marqué dans cet espace national. Cependant, la raison d'être de l'institution est d'accueillir des touristes, et surtout des touristes internationaux. Nous pourrions même suggérer que l'OdT constitue un espace international, ou «espace mondialisé » (Duchêne, 2011), ce qui implique que le choix linguistique non-marqué pourrait s'étendre à d'autres langues que le français. Cette dynamique est reflétée dans le paysage linguistique de l'OdT à travers l'affichage et l'offre de documentations dans 10 langues différentes.

Obligations professionnelles (et touristiques). Bien que toute interaction, et surtout toute interaction de service, soit caractérisée par des objectifs interactionnels, ceux-ci sont particulièrement marqués dans le cadre de l'OdT. Les conseillers ont l'obligation professionnelle de fournir des informations aux touristes. Pour ce faire, ils doivent s'adapter à chaque touriste rapidement, de façon à pouvoir renseigner un grand nombre mais aussi pour que les touristes ne perdent pas de temps dans l'atteinte de leur objectif principal, c'est-à-dire l'entreprise d'activités touristiques. Ces impératifs professionnels créent donc non seulement une obligation d'assurer la réussite de chaque interaction mais également des contraintes temporelles.

Il convient de noter que les cinq phénomènes décrits ici ne sont pas forcément spécifiques aux situations liées au tourisme. En effet, le caractère "fugace» et «tir rapide » des interactions, couplé aux obligations professionnelles, ne sont pas uniques à la situation du tourisme, ils sont également présents dans la quasi-totalité d'interactions de service (cf. Traverso, 2001 ; Filliettaz 2004, par exemple). À cet égard, les interactions en milieu touristique n'en sont qu'un exemple. De la même manière, l'hétérogénéité des provenances et des

\footnotetext{
${ }^{2}$ La durée moyenne d'un échange dans le corpus MITo est 3 minutes et 23 secondes.
} 
répertoires langagiers des locuteurs et l'ambiguïté du contexte sociolinguistique peuvent aussi être observés dans d'autres situations d'interaction plurilingue, telles que l'éducation supérieure et les réunions de travail internationales (Mondada \& Nussbaum, 2012) ou les salons commerciaux internationaux (Piccoli, 2016). Ainsi, en termes de caractéristiques du contexte d'interaction, le tourisme international semble avoir des éléments en commun avec d'autres situations que l'on pourrait qualifier de globalisées. Pour la plupart d'entre-elles, ces caractéristiques sont un produit direct des dynamiques de mobilité globalisée qui constituent le contexte. Il sera intéressant de voir au fil des analyses présentées ici si ces similitudes contextuelles impliquent également des similitudes au niveau du comportement linguistique et interactionnel.

Les cinq facteurs identifiés ici nous permettent donc de relever certains points communs entre le contexte du tourisme international et d'autres situations d'interaction. Cependant, contrairement aux situations d'interaction comparables mentionnées ici, les interactions à l'OdT sont caractérisées quasiment systématiquement par la présence simultanée de ces cinq facteurs. Les interactions sont influencées à la fois par leur caractère « fugace » et « tir rapide », par l'hétérogénéité des locuteurs, par l'ambiguïté sociolinguistique de la situation et par les obligations professionnelles et touristiques. En outre, les interactions en milieu touristique ont un rôle symbolique qui est peut-être plus marqué que dans d'autres contextes : elles sont cruciales dans l'élaboration de l'expérience touristique, et font partie intégrante de celle-ci. Les contextes liés au tourisme international se présentent donc comme des situations particulièrement intéressantes pour l'étude des phénomènes interactionnels et sociolinguistiques.

À l'OdT, comme dans tous les autres contextes mentionnés, il est évident que chaque contrainte interactionnelle constitue un défi pour la communication, rendant le processus d'adaptation langagière entre les locuteurs potentiellement plus complexe. Il est donc impératif de prendre en compte ces contraintes pour comprendre les pratiques langagières à l'OdT, notamment parce qu'elles peuvent compliquer le choix linguistique le plus fondamental pour les interlocuteurs, à savoir: quelle langue utiliser? Dans la section suivante, nous nous consacrons à cette question fondamentale et analysons quelles langues les locuteurs choisissent et comment ils mettent en place ce choix. Ces négociations peuvent potentiellement dévoiler beaucoup d'éléments utiles pour comprendre les dynamiques sociolinguistiques en jeu à l'OdT.

\section{Vers des normes interactionnelles globalisées?}

Le caractère ambigu de l'OdT comme contexte sociolinguistique ouvre potentiellement la possibilité pour les locuteurs d'utiliser d'autres langues que celle qui constitue la norme nationale (le français), amenant ainsi à la création de nouvelles normes linguistiques et interactionnelles. La constitution et l'analyse d'un grand corpus oral tel que le corpus MITo, couplée aux observations sur le terrain, nous permet d'aborder ces normes sous plusieurs angles. Ces analyses, présentées ici, montrent que plusieurs langues sont utilisées de façon récurrente à l'OdT, ce qui suggère que le français, même si elle est la langue majoritaire, ne constitue pas la seule langue légitime (Bourdieu, 2001). Nous montrons comment la fréquence d'utilisation du français et de l'anglais et leur non-marquage dans les discours semblent positionner leur utilisation comme une norme linguistique. Pour ce faire, nous analysons la négociation et la sélection des langues en interaction avant de mettre leur apparente normalisation en lien avec des idéologies linguistiques et sociales plus largement associées à la globalisation. 


\section{Le choix de la langue principale : négociations sélectives}

Les caractéristiques de l'OdT, et surtout l'absence d'histoire interactionnelle, font que les locuteurs ne connaissent pas, en toute probabilité, les préférences linguistiques de leurs interlocuteurs. Ainsi, chaque interaction doit obligatoirement commencer par une négociation pour sélectionner la langue principale de l'échange. Nous appelons ici ces séquences des «négociations sélectives ». Contrairement à ce que l'on pourrait imaginer, il est facile à identifier une (seule) langue principale dans chaque échange. Ainsi, chaque interaction à l'OdT contient une négociation sélective qui conduit au choix de cette langue principale. Bien que le fonctionnement de ces négociations sélectives ne soit pas le point focal de cette étude (cf. Wilson, 2018, pour une analyse détaillée), il semble important de présenter rapidement le déroulement de ces séquences. Dans le corpus MITo, nous observons deux types de négociations sélectives : les demandes explicites et les négociations tacites.

Les demandes explicites sont les négociations dans lesquelles les participants discutent ouvertement et explicitement du choix de code pour l'interaction. Ces demandes thématisent donc la négociation du médium d'interaction et sont présentes dans 12 interactions, soit $13 \%$ du corpus. L'exemple ci-dessous indique une demande explicite dans laquelle les participants se mettent d'accord rapidement sur le médium d'interaction (anglais dans cet exemple). Il s'agit de deux touristes irlandais anglophones (T1 et T2) et d'une conseillère française et francophone $(\mathrm{CF} 6)^{3}$.

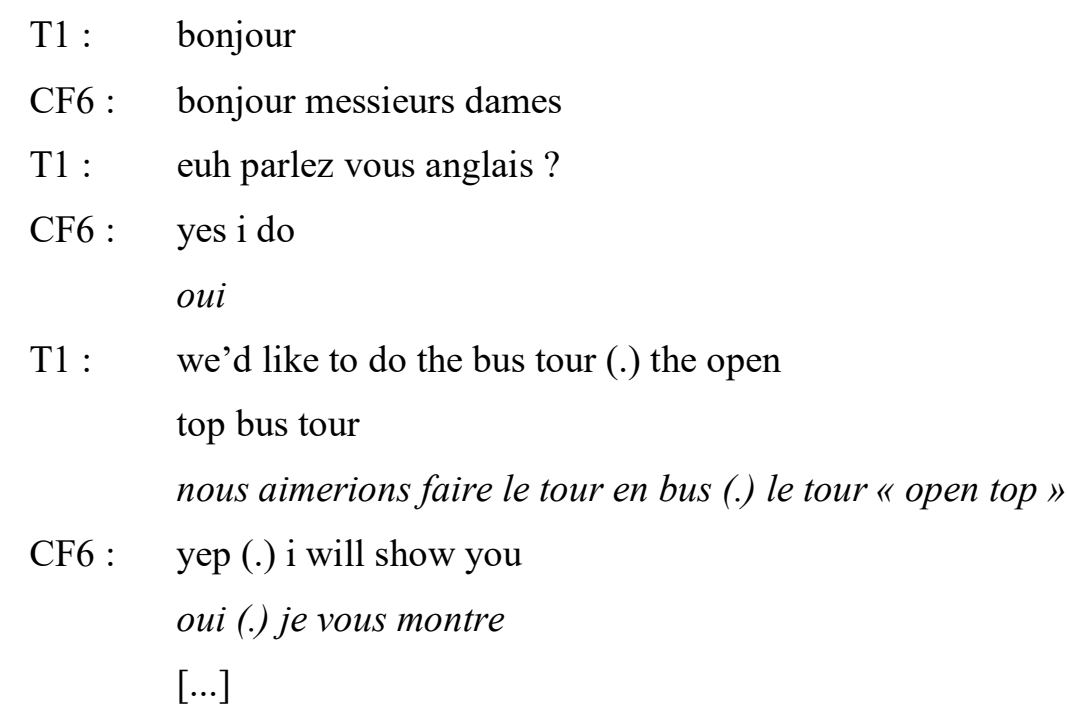

Suite à l'échange de salutations qui se déroule en français, T1 enchaine immédiatement en demandant si CF6 parle anglais. Cette demande est ratifiée non seulement par l'acceptation de CF6 mais également par la ressource linguistique utilisée (l'anglais). Cette réponse est enfin ratifiée et confirmée par $\mathrm{T} 1$ qui formule sa demande d'information en anglais, ce qui mène à l'utilisation de cette ressource pour le reste de l'interaction. Comme dans cet exemple, toutes les demandes explicites sont initiées par des touristes, il n'y a dans le corpus aucune occurrence d'une telle demande de la part d'un conseiller.

Bien que les demandes explicites puissent apparaitre comme le déroulement le plus logique d'une négociation sélective, elles sont relativement rares dans le corpus. Nous observons beaucoup plus de négociations tacites, se caractérisant par la proposition et la ratification (ou pas) d'un médium d'interaction sans que cela soit thématisé. Les négociations tacites sont présentes dans 80 interactions, soit $86 \%$ du corpus.

\footnotetext{
${ }^{3}$ Les conventions de transcription se trouvent en annexe à la fin de cet article.
} 
L'exemple suivant présente le début d'une interaction entre une touriste japonaise (T3) et une conseillère française (francophone) $(\mathrm{CF} 1)$.

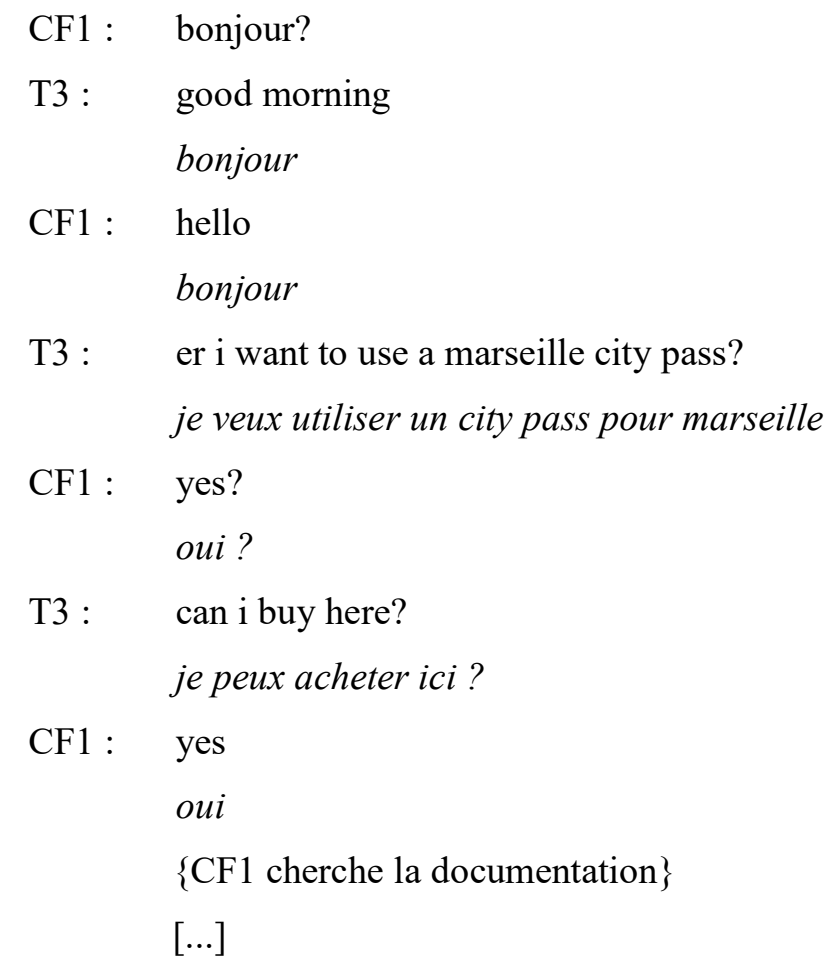

Ici, CF1 débute l'interaction avec une salutation en français. Il convient de noter que, à chaque fois qu'un conseiller prend le premier tour d'une interaction, ce premier tour est initié par un «bonjour » comme nous le voyons ici. Dans cet exemple, T3 répond en anglais avec une salutation comparable, ce qui constitue potentiellement une réponse marquée parce qu'elle répond dans une autre langue. La fonction de ce premier tour de T3 n'est pas seulement de répondre à une salutation mais également de proposer une nouvelle langue principale d'interaction et ainsi de refuser le code sélectionné par CF1. CF1 ratifie implicitement cette proposition dans le tour suivant en répondant avec une salutation en anglais et ainsi établit la langue « de base » pour l'interaction. À aucun moment cette négociation ne fait partie d'une thématisation dans le discours, le processus est accompli de façon tacite.

\section{Des négociations sélectives aux normes interactionnelles}

Le type d'analyse exposée ci-dessus a été mené sur les 93 interactions du corpus MITo, ce qui permet à la fois une analyse fine du comportement langagier dans chaque interaction et une vue d'ensemble des tendances linguistiques (quantitatives) à l'OdT. Un tel protocole nous permet de dégager certaines normes interactionnelles grâce à la quantité importante de données et la granularité des analyses. La première observation qui peut être faite au sujet des normes sociolinguistiques de l'OdT concerne la fréquence de certaines langues dans les interactions. Bien que le corpus MITo soit constitué de 93 interactions dont l'ensemble dure plus de cinq heures, seulement cinq langues sont employées dans les interactions en face-à-face entre les touristes internationaux et les conseillers à l'OdT :

- français (57 interactions, soit $61,3 \%$ du corpus)

- anglais (26 interactions, soit $27,9 \%$ du corpus)

- espagnol (3 interactions, soit $3,2 \%$ du corpus)

- allemand ( 3 interactions, soit 3,2\% du corpus) 
- italien (3 interactions, soit 3,2\% du corpus) ${ }^{4}$

Malgré l'hétérogénéité des répertoires linguistiques des touristes et malgré la présence de plusieurs langues dans le paysage linguistique de l'OdT, les choix linguistiques des locuteurs sont très schématiques, valorisant un nombre restreint de langues et accordant une valeur particulièrement élevée au français et à l'anglais.

La valorisation systématique de certaines langues et le caractère schématique de leur sélection - à travers des négociations tacites ainsi que, à un degré moindre, les demandes explicites - constituent des schémas récurrents de comportement linguistique. Marie-Louise Moreau (1997 : 218) appelle ce type de comportement schématique une "norme objective ", c'est-à-dire des « habitudes linguistiques partagées par les membres d'une communauté ou d'un sous-groupe de celle-ci. Ce sont les règles qui sous-tendent les comportements linguistiques, indépendamment de tout discours méta- ou épilinguistique ». La sélection de l'une des cinq langues citées ci-dessus - et surtout du français ou de l'anglais - par le biais d'une négociation tacite ou d'une demande explicite constitue donc une telle norme objective, observable empiriquement grâce à la grande quantité de données interactionnelles.

Cette analyse quantitative peut être renforcée par l'analyse qualitative du comportement des locuteurs en interaction qui rend compte également du caractère normatif des pratiques observées. En analysant toutes les interactions du corpus, nous observons que le choix des langues les plus fréquentes (le français et l'anglais) n'est jamais remis en question, ni refusé par les locuteurs. Ces choix semblent constituer donc un comportement linguistique nonmarqué et, ainsi, normal. Cette dynamique est visible dans les exemples ci-dessus et également dans le fait que les locuteurs montrent une nette préférence pour les négociations tacites dans lesquelles le choix de langue n'est pas thématisé ou marqué.

Nous observons donc une normalisation du français et de l'anglais dans le contexte de l'OdT à travers le fait que le choix de ces langues ne soit jamais marqué dans les interactions. Bien qu'on observe l'utilisation de l'espagnol, l'allemand et l'italien dans le corpus, il y a au moins une interaction pour chacune de ces langues dans laquelle elles sont refusées par l'un des locuteurs et ainsi marquées comme étant inappropriées, ou « anormales ». À titre d'illustration, l'exemple suivant montre ce qui se passe quand un touriste germanophone (T4) essaie d'initier une négociation tacite avec une conseillère francophone (CF8) en employant une autre ressource que le français ou l'anglais.
CF8 : bonjour?
T4: haben sie ein stadtplan?
avez-vous un plan de la ville?
CF8 : er i don't speak er dutch hein
euh je ne parle pas néerlandais hein
T4 : $\quad$ er a map
euh un plan
CF8 : a map \{rires\}
$\{\mathrm{CF} 8$ cherche la documentation et la donne à
T4\}
T4 : $\quad$ merci

\footnotetext{
${ }^{4}$ Ces chiffres ne tiennent pas compte d'une interaction dans laquelle il est très difficile d'identifier une seule langue principale. Il s'agit de la seule exception du corpus. Les langues employées dans cette interaction sont le français, l'anglais et l'allemand.
} 
CF8 : you're welcome

de rien

T4 répond ici à une salutation en français dans une autre langue, sans formuler une salutation en retour, proposant ainsi une ressource linguistique (l'allemand) qui est différente de celle proposée par la conseillère en ouverture (le français). CF8 rejette cette proposition en disant qu'elle ne parle pas allemand ${ }^{5}$. Cet énoncé constitue un refus clair de la ressource proposée, ce qui est compréhensible étant donné que la conseillère ne parle pas allemand. Cependant, audelà d'un simple refus, l'énoncé marque la proposition de l'allemand comme étant un comportement inattendu dans ce contexte. Cependant, CF8 change de code et, ainsi, en même temps qu'elle refuse l'allemand, elle propose l'anglais comme ressource. T1 ratifie cette proposition sans la thématiser dans son prochain tour en reformulant sa requête en anglais.

L'exemple ci-dessus montre comment certaines langues sont marquées en négociation tandis que d'autres ne le sont pas. Ici, l'allemand est marqué comme étant non souhaitable mais l'absence de marquage concernant l'anglais positionne celui-ci comme une ressource acceptable ou normale, un positionnement consolidé par l'absence de marquage dans la ratification de son utilisation. L'élément clé ici n'est pas le refus de l'allemand en soi mais le caractère non-marqué de la proposition de l'anglais pour pallier les difficultés linguistiques. Le français et l'anglais sont les seules langues qui sont concernées par cette absence de marquage et semblent donc bénéficier d'un statut de langue(s) légitime(s) dans ce contexte.

Ces dynamiques sont également visibles dans l'exemple suivant. Il s'agit d'un échange entre deux touristes italiens (T5 et T6) et une conseillère francophone (CF1) qui a également des compétences en anglais, espagnol et italien.

CF1 : bonjour?

T5 : bonjour (.) vous parlez italien?

CF1 : euh italien (.) or french english

euh italien (.) ou français anglais

T6 : $\quad$ ah (.) euh (.) english

ah (.) euh (.) anglais

CF1 : english?

anglais

\{T5 et T6 entendent une conseillère parler italien au guichet suivant\}

T5 : nous attend

CF1 : oui oui oui si vous voulez

\{T5 et T6 se dirigent vers le guichet voisin\}

Dans cet exemple, T5 formule une demande explicite et propose (en français) l'italien comme langue d'interaction. CF1 répète « italien », ce qui semble constituer une acceptation (bien que dans un médium potentiellement non préféré, c'est-à-dire en français plutôt qu'en italien). Cependant, malgré le fait que CF1 parle italien, elle enchaine à cette réponse une alternance codique vers l'anglais pour proposer le français et l'anglais. Cet énoncé semble

\footnotetext{
${ }^{5}$ Il convient de noter que CF8 utilise le mot «Dutch », désignant « néerlandais » en anglais, pour désigner la langue parlée par T4 (l'allemand). Il semble peu probable que CF8 se soit trompé dans l'identification de la langue de T4. Nous postulons que soit CF8 confond le mot «Dutch» en anglais avec le mot allemand «deutsch», désignant allemand, soit qu'elle fait une erreur de prononciation.
} 
suggérer, contrairement à son énoncé précédent, un refus de la part de CF1 de l'italien. T6 hésite, avant de ratifier l'anglais comme langue d'interaction. Cependant, les deux touristes entendent une autre conseillère parler italien à un autre guichet et décident donc d'y attendre ${ }^{6}$.

Tout comme dans l'exemple précédent, nous observons un non-marquage de l'anglais ou du français juxtaposé à un marquage d'une autre langue (l'italien dans ce cas). Même si la conseillère parle italien, elle montre une préférence claire pour une interaction en anglais ou en français. Plusieurs exemples de ce phénomène ont été observés dans le corpus et sur le terrain de l'OdT pendant les périodes d'observation. Par exemple, un conseiller (français) pouvant parler japonais a été observé plusieurs fois en interaction avec des touristes japonais. Ces interactions se déroulaient systématiquement en anglais et le conseiller en question n'essayait jamais d'initier un changement de langue vers le japonais. De la même manière, malgré l'hétérogénéité extrême des répertoires langagiers des touristes à l'OdT, à aucun moment un touriste n'essaie d'utiliser une langue qui n'est pas le français, l'anglais, l'espagnol, l'allemand ou l'italien. Les raisons pour les « refus » d'utiliser certaines compétences linguistiques, que ce soit à travers des négociations des langues en interaction ou par la réticence à utiliser certaines compétences linguistiques, sont compréhensibles et justifiables. Il peut s'agir par exemple d'une manifestation d'insécurité linguistique ou du respect du choix de langue de l'autre. Cependant, ce qui retient ici notre attention est le fait que ces refus soient couplés au marquage de certaines langues comme étant inutilisables, et donc inacceptables, dans ce contexte. Le français et l'anglais sont les seules langues qui ne sont jamais marquées comme telles dans le corpus ou dans les autres interactions observées lors du travail du terrain à l'OdT.

En somme, une norme linguistique semble pouvoir être dégagée concernant le comportement en interaction à l'OdT : la sélection du français ou de l'anglais comme langue principale d'interaction, choisis très majoritairement par des négociations tacites. Ces normes sont observables empiriquement grâce à leur fréquence dans les données du corpus et le fait que ce comportement ne soit jamais marqué en interaction. Ainsi, malgré les caractéristiques du contexte discutées ci-dessus, nous observons un comportement langagier très schématique. Certaines dynamiques sociales et économiques pourraient aider à la compréhension de ce phénomène.

\section{Dynamiques sociolinguistiques d'efficience}

Dans le contexte globalisé de l'OdT, nous avons identifié certains schémas normatifs de comportement linguistique, malgré les caractéristiques du contexte qui semblaient promouvoir un comportement hétérogène. Pour éclairer cette situation, ces observations peuvent être mises en lien avec des dynamiques socio-économiques plus larges de la globalisation, et notamment avec les idéologies qu'elle propage.

Le sociologue Alain Bihr (2011) explore comment l'idéologie néolibérale de la globalisation se diffuse dans les sociétés, en montrant comment les principes nécessaires au bon fonctionnement du néolibéralisme - tels que l'individualité, l'autodétermination, la compétitivité et la flexibilité, par exemple - deviennent peu à peu des valeurs sociales appréciées et recherchées par des individus. L'une de ces valeurs est celle de l'efficience, c'està-dire un rendement optimal pour un minimum de dépense de ressources ou d'effort. L'efficience, notion économique devenue trait de personnalité, peut être mobilisée afin d'analyser les pratiques langagières à l'OdT. Compte tenu des obligations professionnelles et touristiques introduites précédemment, nous postulons que les locuteurs, comme dans toute interaction de service, opèrent des choix linguistiques dans ce contexte emblématique de la

\footnotetext{
${ }^{6}$ Cet exemple fournit également un exemple de la « réorientation » de clients vers certains employés en fonctions des langues parlées. Faute de place, ce phénomène n'est pas abordé ici mais fera l'objet d'une recherche ultérieure plus approfondie.
} 
globalisation avec l'objectif d'être, avant tout, " efficients » dans leur interaction. La notion d' " efficience » est utilisée ici donc pour caractériser la co-construction du sens entre les participants d'une interaction de la façon la plus rapide possible. Ainsi, une interaction « efficiente » serait un échange au sein duquel les participants réussissent à atteindre l'objectif interactionnel - la transmission d'information - rapidement, sans perdre du temps ou de l'effort ${ }^{7}$. Pour assurer l'efficience d'une interaction, les locuteurs choisissent les ressources linguistiques qu'ils perçoivent comme étant les plus efficaces. Nous postulons ainsi que le français et l'anglais sont valorisés puisqu'ils sont perçus comme étant les ressources les plus « efficientes » dans le contexte de l'OdT $^{8}$.

La perception du français et de l'anglais comme étant des ressources particulièrement efficaces dans ce contexte peut être mise en relation avec des idéologies linguistiques qui concernent ces langues. Plusieurs travaux ont fait état de l'idéologie unilingue qui règne en France (cf. Boyer, 2012, par exemple). Cette idéologie positionne le français comme la seule et unique langue de communication en France, lui accordant ainsi le statut de ressource par excellence pour une interaction efficiente dans un contexte français. Il convient de noter que cette idéologie est extrêmement présente non seulement en France mais également à l'étranger. Si nous concevons l'OdT comme un contexte français, le français s'impose comme la seule option permettant une communication efficiente et son utilisation demeure donc non-marquée et « normale ».

En revanche, si nous considérons l'OdT comme un contexte international, la normalisation de l'anglais peut être mise en lien avec les idéologies qui positionnent l'anglais comme une langue véhiculaire globale. L'expansion globale de l'anglais a été accompagnée d'une expansion concomitante de discours qui le positionnent comme une langue particulièrement adaptée à être utilisée comme une lingua franca globale (cf. Mufwene, 2010). L'anglais est donc positionné comme la langue par excellence pour atteindre la communication " efficiente » dans une situation internationale.

Nous observons donc les traces de deux idéologies linguistiques différentes dans les pratiques langagières à l'OdT : une idéologie «locale » (ou «nationale" dans ce cas) qui valorise le français et une idéologie « globale » qui valorise l'anglais. Ces dynamiques semblent être liées à la recherche d'efficience des locuteurs et la valorisation schématique du français et de l'anglais, ainsi que leur caractère non-marqué, suggère qu'ils sont perçus par les locuteurs qui les emploient comme des langues efficientes et ainsi légitimes dans ce contexte. Dans la quasi-totalité des cas, l'emploi de ces langues a comme résultat une interaction que l'on pourrait juger efficiente. Ainsi, l'expérience des pratiques langagières vécues par les locuteurs à l'OdT contribue à renforcer les idéologies diffusées par d'autres canaux dans la société.

À travers l'étude du corpus MITo et le travail de terrain à l'OdT, nous avons pu identifier certains schémas récurrents de comportement langagier. Malgré l'hétérogénéité des répertoires langagiers présents à l'OdT, les locuteurs valorisent un petit nombre de langues en interaction. Parmi ces langues, le français et l'anglais sont particulièrement valorisés. Le choix de ces langues se fait à travers les négociations sélectives et les locuteurs montrent une préférence considérable pour les négociations tacites. La fréquence avec laquelle ces langues sont choisies et le fait que leur sélection reste quasiment toujours non-marquée en interaction suggèrent que la sélection et l'utilisation du français et/ou de l'anglais constituent une norme linguistique dans ce contexte. Ces normes peuvent être éclairées en faisant référence à la diffusion de l'efficience en tant que valeur fondamentale au sein de la globalisation et aux idéologies linguistiques qui

\footnotetext{
${ }^{7}$ Cette définition de la communication «efficiente» est clairement discutable et limitée mais nous semble pertinente étant donné l'accent mis sur la transmission d'information à la fois par les conseillers (et leurs supérieurs) et les touristes.

${ }^{8}$ Il ne s'agit pas de suggérer que le français et l'anglais constituent des ressources particulièrement « efficaces » sur le plan linguistique mais bien qu'ils sont perçus comme étant plus efficaces par les locuteurs.
} 
positionnent le français et l'anglais comme étant particulièrement efficaces pour la communication dans un contexte tel que l'OdT. Nous observons donc comment les pratiques contextualisées de l'OdT peuvent être mises en relation avec les dynamiques socioéconomiques plus larges de la globalisation.

L'omniprésence de ces idéologies linguistiques et culturelles nous aide à rationaliser un comportement uniforme dans un contexte hétérogène. Cependant, nous pouvons nous interroger sur l'apparition de normes linguistiques aussi fortes dans ce contexte, étant donné que de telles normes ont tendance à se développer à l'intérieur d'une communauté, tandis que les locuteurs à l'OdT ne se connaissent pas.

\section{Communauté de pratique discontinue}

Le caractère récurrent des pratiques, l'abondance de négociations tacites, et la fugacité des interactions suggèrent que les participants à l'OdT «savent» déjà comment « il faut se comporter » linguistiquement, même s'ils ne se connaissent pas et ne partagent aucune histoire interactionnelle ensemble. Autrement dit, ils semblent avoir développé une conscience normative pour cette situation, même s'ils ne l'ont jamais vécue auparavant. Pour essayer d'expliquer cette prise de conscience normative sans contact préalable, nous proposons ici d'explorer, et étendre, la notion de « communauté de pratique ».

La notion de communauté linguistique, ou des notions similaires sous d'autres noms, ont fait l'objet de beaucoup de discussion en sociolinguistique (cf. Bretegnier, 2017, par exemple). Il est au-delà du champ de cet article de rentrer dans ces discussions mais une notion nous semble particulièrement pertinente afin d'éclairer la prise de conscience normative observée à l'OdT : la notion de «communauté de pratique » comme définie par Penelope Eckert (2006). Eckert définit une communauté de pratique comme un «ensemble de personnes qui s'engagent de façon récurrente dans une entreprise commune quelconque ${ }^{9}$ (Eckert, $2006: 683$ ). À travers un accent mis sur les pratiques partagées d'un groupe de personnes, Eckert démontre comment cette pratique commune récurrente génère des façons de faire et des façons de dire propres à la communauté et ainsi développe un sens sociolinguistique pour ces comportements. Pour que ce sens soit "conventionnalisé », deux conditions sont nécessaires : un engagement pour la compréhension commune et une expérience partagée dans le temps.

À l'OdT, il est clair que les locuteurs participent à un engagement pour la compréhension commune : ils utilisent des stratégies de communication afin d'assurer la compréhension entre les interlocuteurs en interaction. En outre, ils s'engagent à élaborer ensemble une compréhension de l'activité en cours, c'est-à-dire un échange d'informations touristiques dans lequel certains participants endossent l'identité de touriste et d'autres de conseiller (et, par extension, de « local »). Bien que ce critère pour la « conventionnalisation » du sens soit rempli, le deuxième critère pose problème ici : les interlocuteurs ne peuvent pas avoir une expérience partagée dans le temps puisqu'ils n'ont aucune histoire interactionnelle ensemble (et n'en auront pas). Cependant, nous observons une schématisation du comportement à l'OdT qui suggère le développement de façons de parler et d'agir qui rappellent celles d'une communauté de pratique.

Nous proposons d'étendre la définition de communauté de pratique en y ajoutant la notion de discontinuité. Même si un locuteur à l'OdT n'a aucune histoire interactionnelle avec les autres locuteurs présents, il est facile d'imaginer qu'il a expérimenté des situations très similaires (d'autres situations touristiques, par exemple) à plusieurs reprises dans sa vie, bien qu'avec d'autres interlocuteurs. De la même façon, malgré la diversité des touristes renseignés à l'OdT, les besoins sont très similaires d'un touriste à l'autre, ce qui crée un caractère répétitif

\footnotetext{
${ }^{9}$ « a collection of people who engage on an ongoing basis in some common endeavor ».
} 
dans le travail des conseillers. Ces constats rappellent l'idée selon laquelle l'histoire interactionnelle d'un individu est «la totalité des interactions auxquelles il a participé ou assisté » (Vion, 1992 : 99) dans sa vie. Nous suggérons donc que tous les locuteurs à l'OdT s'engagent bel et bien dans une expérience partagée dans le temps, mais que cette expérience est partagée avec des interlocuteurs différents, dans des lieux et à des moments différents. Nous formulons cette idée par l'expression de « communauté de pratique discontinue » : c'est-à-dire une communauté fondée sur un partage de normes acquises à travers une participation dans une entreprise commune, répétée régulièrement dans les trajectoires de vie de chacun, bien que presque jamais avec les mêmes interlocuteurs. La communauté d'acteurs formée par les touristes rassemble donc des flux d'individus mobiles, qui se côtoient de façon fugace, ou éphémère, participent à des situations à la fois identiques et différentes, hétérogènes et homogènes. Chaque interaction contextualisée est influencée par un nombre incalculable d'autres interactions, qui ont parfois lieu dans des contextes géographiquement très éloignés. Cette interconnexion crée le réseau de la communauté de pratique discontinue.

La notion de communauté de pratique discontinue suppose que les normes linguistiques soient à priori très similaires dans l'ensemble des situations semblables vécues par les locuteurs. Dans le cas étudié ici, il s'agirait de supposer que les normes linguistiques observables à l'OdT seraient observables dans d'autres contextes touristiques. De cette manière, l'OdT constituerait une brique dans la construction d'une conventionnalisation du sens et ainsi dans le développement de normes liées aux contextes touristiques. L'étude présentée ici se focalise seulement sur un seul événement dans ce processus; par conséquent l'existence d'une communauté de pratique discontinue ne peut pas être vérifiée empiriquement. Cependant, le caractère hautement schématique des normes linguistiques observées à l'OdT suggère une socialisation importante à des situations présentant les mêmes normes. Il nous semble que la communauté de pratique discontinue constitue une piste intéressante pour les recherches futures puisque cela nous permettrait d'envisager comment les interactions contextualisées, occasionnelles et exceptionnelles peuvent être mises en relation avec les dynamiques sociales plus larges.

Nous pourrions même aller plus loin avec cette notion, en formant l'hypothèse que les schémas linguistiques observés à l'OdT s'appliquent non seulement à d'autres lieux touristiques mais à d'autres «situations clés» de la globalisation (ou, en tout cas, de la globalisation des privilégiés). Pour ne prendre qu'un exemple, nous pourrions faire le rapprochement entre les normes linguistiques observées à l'OdT et les données présentées par Piccoli (2016) dans une étude de salons commerciaux internationaux. Bien que Piccoli observe plus d'interactions plurilingues que ce que nous avons observé à l'OdT, le choix des langues utilisées au sein de ces échanges rappelle les schémas de l'OdT : les locuteurs privilégient l'anglais, les langues nationales des pays dans lesquels les locuteurs se trouvent (en l'occurrence le français ou l'italien) ainsi que l'espagnol, une autre grande langue romane. L'entreprise partagée par la communauté de pratique discontinue ne se limiterait donc pas au tourisme mais engloberait d'autres domaines emblématiques auxquels ces acteurs, en toute probabilité, participent. Comme nous l'avons vu, ils se retrouvent donc face à des caractéristiques similaires en termes de contexte d'interaction, et à des normes linguistiques communes.

Nous voyons donc comment la globalisation nous met tous en contact tout en nous gardant éloignés les uns des autres, comment elle crée une communauté formée d'individus qui ne se connaissent pas. Cette observation nous mène à porter une réflexion sur les répercussions sociales de ces dynamiques. 


\section{Répercussions sociales des pratiques langagières globalisées}

Les normes interactionnelles observées à l'OdT, mises en lien ici avec une communauté de pratique discontinue, reflètent des bouleversements au niveau du marché linguistique, ayant des répercussions sociales concrètes.

Tout d'abord, il semble important de souligner le fait que les schémas de comportement linguistique observés à l'OdT constituent une manifestation importante d'inégalité. Nous observons une forte valorisation d'un nombre restreint de langues "globales » (qui sont, par ailleurs, des langues principales des puissances dominantes de la globalisation), au profit de toute autre ressource linguistique. Cette inégalité semble aller à l'encontre des discours très répandus qui mettent en valeur le plurilinguisme à l'ère de la globalisation. De tels discours sont même présents au sein de l'OdT, qui promeut explicitement l'accueil des touristes dans « leurs langues ». Paradoxalement, la promotion d'une pluralité linguistique semble produire l'effet inverse. Il s'agit d'un reflet sociolinguistique du mythe de la théorie de ruissellement ${ }^{10}$ au cœur de la globalisation : au lieu de mener à une égalisation, la promotion d'une égalité entre langues, c'est-à-dire la dérégulation des marchés linguistiques, semble accorder encore plus de poids aux langues dominantes.

Ces dynamiques ont des répercussions profondes pour un grand nombre de personnes. La normalisation de l'utilisation quasi exclusive de certaines ressources linguistiques dans un certain contexte social crée des enjeux d'accès et d'exclusion : seulement ceux disposant de ces ressources dans leurs répertoires peuvent y accéder. Nous retrouvons cette situation à l'OdT car, pour « être » un touriste, il faut parler « comme un touriste », et cela se fait notamment par l'emploi des ressources valorisées décrites dans cet article. Cette dynamique est normative dans le sens où elle prescrit un certain répertoire comme plus acceptable que des autres.

Il en va de même pour les conseillers. La «part langagière » (Boutet, 2001) constitue la quasi-totalité de leur travail. Ainsi, le répertoire langagier d'un individu joue un rôle central dans l'accès à ce milieu professionnel : il est nécessaire de maitriser les ressources valorisées pour pouvoir entrer dans ce marché de travail. D'autres ressources constituent un «bonus », mais le français et l'anglais demeurent "nécessaires ». Une compétence dans ces langues devient donc un prérequis pour accéder à ce milieu professionnel, au même titre que des diplômes et des expériences professionnelles. Les langues, valorisées selon une dynamique extrêmement inégalitaire, jouent donc le rôle d'un "gardien » qui permet à certains d'entrer dans ce milieu et d'en bénéficier tout en fermant l'accès aux autres.

La normalisation des pratiques décrite dans cet article contribue à «cacher» ces enjeux d'inégalité et d'exclusion. L'adhésion à ces normes semble aller de soi, démontrable à travers l'absence presque totale de thématisation du comportement. Cette normalisation contribue également à la production de ces enjeux. Les participants semblent connaitre les normes et, en y adhérant, ils les (re)produisent, et de fait (re)produisent ainsi les dynamiques d'inégalité et d'exclusion qui y sont liées, bien que ce lien soit invisible pour les locuteurs.

Les dynamiques d'inégalité et d'exclusion illustrent comment les pratiques langagières d'un contexte comme l'OdT peuvent être reliées à des dynamiques sociales plus larges. Les dynamiques linguistiques de l'OdT sont à la fois les produits d'une situation caractéristique de la globalisation et des éléments qui contribuent aux dynamiques plus larges de la globalisation. Produites par des locuteurs mobiles dans un cadre de consommation et de commodification, les pratiques langagières renforcent les phénomènes d'inégalité et d'exclusion observés dans d'autres domaines globalisés.

Nous avons déjà noté que certains aspects des schémas linguistiques observés ici peuvent aussi être observés dans d'autres situations globalisées. Cependant, d'autres pratiques

\footnotetext{
${ }^{10}$ L'idée selon laquelle les revenus des plus riches sont réinjectés dans la société, contribuant à l'activité de l'économie plus généralement et profitant ainsi à tous les membres d'une société.
} 
observées à l'OdT se distinguent nettement de celles observés par d'autres chercheurs dans d'autres contextes globalisés : la superdiversity dans les villes globales, le translanguaging dans des classes plurilingues ou le metrolingualism dans des marchés urbains, par exemple. Ces pratiques plurilingues émergeantes contrastent avec la valorisation systématique de grandes langues mondiales dans des interactions généralement monolingues à l'OdT. Loin de nous pousser à remettre en question les notions de superdiversity, metrolingualism, translanguaging ou autres, cette observation nous mène à interroger les différents processus de globalisation qui sous-tendent chaque situation.

Les pratiques des touristes sont celles d'un groupe de personnes qui bénéficient de la globalisation, mobiles pour le plaisir, l'élite de l'expansion du néolibéralisme. Inversement, les pratiques étudiées relevant de la superdiversity, du metrolingualism, ou du translanguaging émergent dans des contextes moins favorisés, au sein de populations qui se trouvent en mobilité pour survivre, avec des individus qui sont donc plutôt en marge de la globalisation. Ces regroupements, tous deux emblématiques de la globalisation, entrent en résonance avec les « deux globalisations » proposées par Zygmunt Bauman (1998): la globalisation des « touristes », les élites qui sont mobiles par choix, et la globalisation des « vagabonds », ceux qui sont mobiles par défaut de choix. Nous proposons ici l'idée que ces " deux globalisations » entrainent des pratiques langagières fortement différentes. Ainsi, les pratiques de type « superdivers » ${ }^{11}$ se développent chez les « vagabonds », tandis qu'un répertoire restreint de langues globales règne chez les « touristes ». Même si la globalisation se présente comme une chance d'unification, d'égalisation et d'harmonisation pour tous, il apparait que l'effet produit est bien le contraire, divisant les populations en deux groupes : " gagnants » et « perdants » de la globalisation.

\section{Conclusion}

L'objectif de cet article était de décrire et analyser des pratiques langagières dans un cadre emblématique de la globalisation : l'Office de Tourisme de Marseille. À travers des analyses quantitatives et qualitatives de données interactionnelles, couplées à un travail de terrain ethnographique, nous avons identifié des normes linguistiques dans ce contexte qui peuvent être mises en lien non seulement avec d'autres contextes globalisés mais aussi avec certaines dynamiques économiques et sociales de la globalisation. Plus précisément, la prédominance du français et de l'anglais dans ce contexte peut être liée à une recherche d'efficience, valeur centrale aux discours et idéologies néolibéraux de la globalisation. Nous avons proposé la notion de « communauté de pratique discontinue » afin d'expliquer l'apparent développement d'une conscience normative au sein d'un groupe de locuteurs qui n'ont aucune histoire interactionnelle partagée. Enfin, nous avons exploré quelques répercussions sociales de ces dynamiques sociolinguistiques, qui touchent non seulement l'OdT ou le tourisme mais tous les individus concernés par les processus de la globalisation.

Pendant l'élaboration de ce travail, nous avons souvent entendu des commentaires qui suggéraient que le comportement linguistique observé à l'OdT n'était ni surprenant, ni inattendu. L'usage prédominant du français et de l'anglais est perçu comme un phénomène tout à fait naturel ou banal, et ainsi vide d'intérêt pour la sociolinguistique. Cependant, bien que normal dans le sens où nous avons l'habitude de le voir dans des situations comparables, nous argumenterions que ce phénomène est à la fois remarquable et complexe sur le plan sociolinguistique. Dans un cadre caractérisé par autant d'hétérogénéité de locuteurs et de

\footnotetext{
${ }^{11}$ Nous entendons ici par « superdivers » des pratiques langagières caractérisées par des alternances et mélanges codiques fréquentes et variées et dans lesquelles nous trouvons des traces des parcours migratoires des locuteurs et leurs communautés (cf. Arnaut et al., 2015).
} 
répertoires langagiers, l'existence de schémas de comportement linguistique fortement homogènes est, selon nous, remarquable. De plus, les pratiques langagières observées à l'OdT sont caractérisées par leur complexité : la co-construction du sens entre un conseiller français et un touriste chinois par le biais de l'anglais utilisé comme langue véhiculaire n'a rien de simple. Plutôt que banal, naturel ou sans intérêt, il s'agit selon nous d'un ensemble de pratiques complexes, avec des enjeux complexes, qui ont été normalisés. La globalisation est remplie d'exemples de phénomènes remarquables, entrainant des conséquences parfois très graves, mais représentés comme normaux et anodins : le client européen qui commande un ordinateur conçu aux États-Unis, fabriqué dans des conditions déplorables en Asie à partir de matières premières récoltées dans des conditions encore plus abjectes en Afrique, et qui voit son produit livré à sa porte. Ces pratiques sont simultanément remarquables et complexes, mais considérées comme normales, anodines et vides d'intérêt pour la grande majorité de la population, malgré les effets secondaires désastreux qu'elles peuvent avoir. À leur échelle, les pratiques langagières à l'OdT fournissent un autre exemple de ces dynamiques. La puissance de ces pratiques est, justement, dans leur normalité. Nous ne pouvons qu'insister sur l'importance de plus de recherche dans des situations emblématiques de la globalisation afin d'explorer davantage ces phénomènes «banals » qui entrainent des conséquences incalculables, que ce soit au niveau linguistique ou social, au sein du contexte en question ou parfois dans des contextes (beaucoup) plus éloignés.

\section{Bibliographie}

ARNAUT Karel, BLOMMAERT Jan, RAMPTON Ben \& SPOTTI Massimiliano, 2015, Language and Superdiversity. Routledge, New York.

AUZANNEAU Michelle \& TRIMAILLE Cyril, 2017, «L'odyssée de l'espace en sociolinguistique », Langage et société, $\mathrm{n}^{\circ}$ 160-161(2), pp. 349-367.

BAUMAN Zygmunt, 1998, Globalization: The Human Consequences, Columbia University Press, New York.

BIHR Alain, 2011, «L'idéologie néolibérale », Semen. Revue de sémio-linguistique des textes et discours, $\mathrm{n}^{\circ} 30$, pp. 43-56.

BLOMMAERT Jan, 2010, The Sociolinguistics of Globalization, Cambridge University Press, Cambridge.

BOURDIEU Pierre, 2001, Langage et pouvoir symbolique, Seuil, Paris.

BOUTET Josiane, 2001, «La part langagière du travail : bilan et évolution », Langage et société, ${ }^{\circ} 98$, pp. 17-42.

BOUTET Josiane, 2008, La vie verbale au travail - Des manufactures aux centres d'appels, Octares Editions, Toulouse.

BOYER Henri, 2012, "Idéologie sociolinguistique et politiques linguistiques « intérieures » de la France », Synergies, Pays germanophones, n ${ }^{\circ}$, pp. 93-105.

BRETEGNIER Aude, 2017, " "Communauté linguistique" : renoncer ou problématiser », La Bretagne Linguistique, $\mathrm{n}^{\circ} 21$.

CANUT Cécile, DANOS Felix, HIM-AQUILLI Manon \& PANIS Caroline, 2019, Le Langage, une Pratique Sociale. Éléments d'une Sociolinguistique Politique, Presses Universitaires de Franche-Comté, Besançon.

COHEN Erik \& COOPER Robert L., 1986, «Language and tourism », Annals of Tourism Research, $\mathrm{n}^{\circ} 13(4)$, pp. 533-563.

COUPLAND Nikolas, 2010, The Handbook of Language and Globalization, John Wiley \& Sons, Chichester. 
DANN Graham, 1996, The Language of Tourism: A Sociolinguistic Perspective, CAB International, Wallingford.

DE STEFANI Elwys \& MONDADA Lorenza, 2014, «Reorganizing Mobile Formations: When "Guided" Participants Initiate Reorientations in Guided Tours », Space and Culture, $\mathrm{n}^{\circ} 17(2)$, pp. 157-175.

DUCHÊNE Alexandre, 2011, «Néolibéralisme, inégalités sociales et plurilinguisme: l'exploitation des ressources langagières et des locuteurs », Langage et société, $\mathrm{n}^{\circ} 136$, pp. 81-108.

DUCHÊNE Alexandre, 2012, «Des langues, des locuteurs et des marchés : la variabilité de l'appropriation économique de la diversité linguistique », Sociolinguistica, n²6(1), pp. 120-135.

DUCHÊNE Alexandre \& HELLER Monica, 2012, Language in Late Capitalism: Pride and Profit, Routledge, New York.

ECKERT Penelope, 2006, "Communities of Practice». Dans Keith Brown (Éd.), Encyclopedia of Language \& Linguistics (Second Edition), Elsevier, Boston, pp. 683-685.

FILLETTAZ Laurent, 2004, «Le virage actionnel des modèles du discours à l'épreuve des interactions de service », Langage et société, $\mathrm{n}^{\circ} 107-1$, pp. 31-54.

GARCIA Ofelia \& WEI Li, 2013, Translanguaging: Language, Bilingualism and Education. Palgrave Macmillan, Londres.

GUMPERZ John J., 1989, Engager la conversation: Introduction à la sociologie interactionnelle, Les Editions de Minuit, Paris.

HELLER Monica, 2003, Éléments d'une sociolinguistique critique, Didier, Paris.

HELLER Monica, 2010, «The Commodification of Language», Annual Review of Anthropology, n³9(1), pp. 101-114.

HELLER Monica, PUJOLAR Joan, DUCHÊNE Alexandre, 2014, «Linguistic commodification in tourism », Journal of Sociolinguistics, ${ }^{\circ} 18(4)$, pp. 539-566.

INDA Jonathan X. \& ROSALDO Renato, 2007, The Anthropology of Globalization: A Reader, 2nd Edition, Wiley-Blackwell, Malden.

JAWORSKI Adam \& THURLOW Crispin, 2009, " Gesture and movement in tourist spaces », dans Carey Jewitt (Éd.), The Routledge Handbook of Multimodal Analysis, Routledge, New York, pp. 253-262.

JAWORSKI Adam \& THURLOW Crispin, 2010a, « Language and the Globalizing Habitus of Tourism: Toward A Sociolinguistics of Fleeting Relationships » dans Nikolas Coupland (Éd.), The Handbook of Language and Globalization, John Wiley \& Sons, Chichester, pp. 255-286.

JAWORSKI Adam \& THURLOW Crispin, 2010b, Tourism Discourse. Language and Global Mobility, Palgrave-Macmillan, Londres.

KELLY-HOLMES Helen., \& PIETIKÄINEN Sari, 2014, « Commodifying Sámi culture in an indigenous tourism site $»$, Journal of Sociolinguistics, $n^{\circ} 18(4)$, pp. 518-538.

LECLER Romain, 2013, Sociologie de la mondialisation, La Découverte, Paris.

MONDADA Lorenza \& NUSSBAUM Luci, 2012, Interactions cosmopolites : L'organisation de la participation plurilingue, Lambert-Lucas, Limoges.

MOREAU Marie-Louise, 1997, Sociolinguistique: les concepts de base, Éditions Mardaga, Sprimont.

MUFWENE Salikoko, S., 2010, Globalization, Global English, and World English(es): Myths and Facts, dans Nikolas Coupland (Éd.), The Handbook of Language and Globalization, John Wiley \& Sons, Chichester, pp. 29-55.

MYERS-SCOTTON Carol, 1993, Social Motivations for Codeswitching: Evidence from Africa, Clarendon Press, Oxford. 
PENNYCOOK Alastair, \& OTSUJI Emi, 2015, Metrolingualism: Language in the City, Routledge, New York.

PERALDI Michel, DUPORT Claire \& SAMSON Michel, 2015, Sociologie de Marseille, La Découverte, Paris.

PICCOLI Vanessa, 2016, " "Puedes hablar italiano" : négocier la conversation plurilingue dans un salon commercial international »,Domínios de Lingu@gem, n¹0-4, pp. 1326-1348.

PLIHON Dominique, 2007, "La globalisation financière », dans Christian Chavagneux, Françoise Milewski, Jean Pisani-Ferry, Dominique Plihon, Michel Rainelli, \& JeanPierre Warnier (Éds.), Les enjeux de la mondialisation, La Découverte, Paris, pp. 22-37.

SCHOLTE Jan Aart, 2002, "What is globalization? The definitional issue-again », CSGR Working Paper, $\mathrm{n}^{\circ} 109 / 02$. URL : http://wrap.warwick.ac.uk/2010 [consulté le 28/05/2019].

SCHOLTE Jan Aart, 2005, «The sources of neoliberal globalization », URL: http://unrisd.org/UNRISD/website/document.nsf/ab82a6805797760f80256b4f005da1a b/9e1c54ceeb19a314c12570b4004d0881/\$FILE/scholte.pdf [consulté le 28/05/2019].

SELIM Monique, 2004, "Globalisation: consonances et dissonances anthropologiques », Socio-anthropologie, $\mathrm{n}^{\circ} 14$, URL : http://socio-anthropologie.revues.org/386 [consulté le 28/05/2019].

THURLOW Crispin, \& JAWORSKI Adam, 2011, «Tourism discourse: Languages and banal globalization », Applied Linguistics Review, n², pp. 285-312.

THURLOW Crispin, \& JAWORSKI Adam, 2013, "Visible-invisible: the social semiotics of labour in luxury tourism », dans Thomas Birtchnell \& Javier Caletrío (Éds.), Elite Mobilities, Routledge, New York, pp. 176-193.

TRAVERSO Véronique, 2001, «Interactions ordinaires dans les petits commerces : éléments pour une comparaison interculturelle », Langage et société, n $95-1$, pp. 5-31.

URRY John, 2007, Mobilities, Polity, Cambridge.

VION Robert, 1992, La communication verbale, analyse des interactions, Hachette, Paris.

VITORIO, Raymund V. M., 2014, Inequality, mobility, and super-diversity: Linguistic ideologies and performances in the Philippine tourism industry, Mémoire de Master (M2), National University of Singapore, Singapour.

WILSON Adam, 2016, Dynamiques sociolinguistiques de la globalisation: l'exemple de l'Office du Tourisme de Marseille, Thèse de doctorat, Aix-Marseille Université, Aixen-Provence.

WILSON Adam, 2018, « Marseille en V.O. ? La négociation des langues dans des interactions entre touristes internationaux et conseillers touristiques ", dans Ulrich Ammon \& Marcella Costa (Éds.), Sociolinguistica vol. 32 - Language choice in Tourism - with the Focus on Europe, De Gruyter, pp. 103-116.

\section{Conventions de transcription}

(.) pause

\{\} commentaire du chercheur

? intonation montante

italique traduction 


\section{GLOTTOPOL}

Revue de sociolinguistique en ligne

Comité de rédaction : Michaël Abecassis, Salih Akin, Sophie Babault, Claude Caitucoli, Véronique Castellotti, Régine Delamotte, Robert Fournier, Stéphanie Galligani, Emmanuelle Huver, Normand Labrie, Foued Laroussi, Benoit Leblanc, Fabienne Leconte, Gudrun Ledegen, Danièle Moore, Clara Mortamet, Alioune Ndao, Isabelle Pierozak, Gisèle Prignitz.

Rédactrice en chef : Clara Mortamet.

Comité scientifique : Claudine Bavoux, Michel Beniamino, Jacqueline Billiez, Philippe Blanchet, Pierre Bouchard, Ahmed Boukous, Pierre Dumont, Jean-Michel Eloy, Françoise Gadet, Monica Heller, Caroline Juilliard, Jean-Marie Klinkenberg, Jean Le Du, Marinette Matthey, Jacques Maurais, Marie-Louise Moreau, Robert Nicolaï, Didier de Robillard, Paul Siblot, Claude Truchot, Daniel Véronique.

\section{Comité de lecture pour ce numéro :}

Mickael Abecassis, Michelle Auzanneau, Salih Akin, Céline Amourette, Gabriel Bergounioux, Philippe Boula de Mareuil, Catherine Brissaud, Aude Bretegnier, Maria Candea, Véronique Castellotti, Régine Delamotte, Jean-François De Pietro, Catherine Delarue-Breton, Anne Dister, Alexandre Duchêne, Valentin Feussi, Françoise Gadet, Stéphanie Galligani, François Gaudin, Monica Heller, Emmanuelle Huver, Caroline Juilliard, Abou Bakri Kebe, Jean-Marie Klinkenberg, Christian Lagarde, Foued Laroussi, Fabienne Leconte, Evelyne Lloze, Nolwenn Lorenzi, Gudrun Ledegen, Nadja Maillard, Marinette Matthey, Bruno Maurer, Véronique Miguel Addisu, Saskia Mugnier, Claire Oger, Marielle Rispail, Richard Sabria, Véronique Traverso, Christel Troncy, Cécile Van den Avenne. 